\title{
Multi-view Face Detection under Unconstrained condition
}

\author{
Ming $\mathrm{Li}^{\mathrm{a}}$ and $\mathrm{YunYu} \mathrm{Xu}$ \\ School of Computer and Communication, Lanzhou University of Technology, Lanzhou 730050, \\ China. \\ alim3076@163.com, buyunyu1@163.com
}

Keywords: face detection, RBM, DBN, FloatBoost, pyramid architecture.

\begin{abstract}
Multi-view face detection is the main problem we consider in this paper. There has been significant research on this problem, while current state-of-the-art algorithms for this task leave something to be desired. In this paper, we present an algorithm to enhance the face detection performance. Before images were put into the deep belief nets (DBN) model, they were preprocessed in turn, and then capitalized on DBN to train the data automatically. Use FloatBoost (FB) Algorithm to learn these feature, and classify them based on pyramid architecture. Evaluations on dataset show that our proposed algorithm has similar or better performance compared to the current methods. It can accurately spot faces at multi-view, even when partially occluded.
\end{abstract}

\section{Introduction}

Face detection has a wide use in the field of defense and security, video surveillance and human-computer interaction, but the current detection accuracy and detection speed in face detection is difficult to meet the daily life due to various poses, occlusions etc. Specially, poses affect detection accuracy much. The research shows that more than $75 \%$ face images are non-positive [1]. The research focused on the multi-view face detec4tion has become a hotspot.

Various poses introduce nonlinear factors to face detection, thus increase the difficulty of face detection. The current state-of-the-art algorithms always combine several methods to implement face detection [2]. Although these algorithms can improve the performance of face detection to some extent, the discrimination of face features extracted by these algorithms are not strong, the expression of the feature is over dependent on artificial selection, which makes researchers feel confused about how to choose and express accurately. DBN [3] is a typical deep learning model, which was first proposed by Hinton et al. in 2006. It learns abstract features in the different levels top-down, and then obtains the nonlinear description from features, which expresses an automatic feature extraction process. It has been successfully used in image dimension reduction, image recognition $[4,5]$ etc. At present, the softmax algorithm is widely used in DBN model to classify, while it is effective only if the categorization labels are mutual exclusion (take paper [4] for example). In multi-view face detection, faces in each poses scope are highly similar, thus the classification performance will decrease rapidly as we use softmax. FB algorithm [1], which was put forward by Li et al as the improvement of Adaboost, has an illumination and rotation invariance, It not only solves the problem that Adaboost is easy to fall into the endless loop caused by non monotonicity factors, but eliminates the weaker feature on the dataset. In this paper, we combine the two algorithms to provide a more scientific theoretical basis for face detection.

In this paper, we capitalized on the advance made in recent years on a type of deep learning known as a DBN to extract face features automatically on the basis of relevance between each part on face. FB algorithm is used to learn detector of different head rotation. Combine these detectors as a detector-pyramid structure (see for fig.4). Input the features extracted by DBN into this structure, then classify whether there are faces in the image. The experiment results show that our algorithm can accurately spot face under unconstrained. 


\section{Face detection system}

The face detection is to traverse and search any given image or image sequence according to a certain method to determine whether there are faces in the picture, if there are, then spot the face and locate it using a red border marker. In this paper, first use the DBN to extract the feature of human face, and second detector-pyramid architecture as classifier to spot human faces in the images. The face detection framework we designed is shown as the following figure:



Fig. 1 our face detection framework

In this paper, we use DBN to make the system not independent on the artificial selection and automatically learn the abstract features from the input data. The framework of DBN is shown in fig.3. A typical DBN can be regarded as a highly sophisticated directed acyclic graph composed by multiple random variables, which is a superposition of many RBM [5] (see for example fig.2), constituted by bottom-up combining these RBM. For a DBN with $l$ layers of hidden units, the joint probability distribution between the visual units and the hidden units can be expressed as follow:

$$
p\left(v, h^{1}, h^{2}, \ldots, h^{l}\right)=P\left(v \mid h^{1}\right) P\left(h^{2} \mid h^{1}\right) \ldots P\left(h^{l-1}, h^{l}\right)
$$

Where $v=h^{0}, v$ is the visual units, $h^{k}(k=1,2, . l)$ is the hidden units on the $k t h$ layer. The probability between the hidden units in the $k$ th layer and the hidden units in the $k+1$ th layer are:

$$
P\left(h^{k} \mid h^{k+1}\right)=\prod_{i} P\left(h_{i}^{k} \mid h^{k+1}\right) \quad P\left(h_{i}^{k}=1 \mid h^{k+1}\right)=\delta\left(b_{i}^{k}+\sum_{j} w_{i j}^{k} h_{j}^{k+1}\right)
$$

Where $\delta(x)=1 /[1+\exp (-x)]$ is the logistic sigmoid function, $h_{i}^{k}=1$ denotes that the $i$ th unit is visible in the $k$ th layer, $b_{i}^{k}$ is the unbiased sample. ${ }^{w_{i j}^{k}}$ is the weight between the hidden units in the $k$ th layer and the hidden units in the $k+1$ th layer. In this DBN, we regard $p\left(h^{l-1}, h^{l}\right)$ as a RBM model. The energy of the RBM is given as follow:

$$
E(v, h)=-v^{T} w h-v^{T} b^{k-1}-h^{T} b^{k}
$$

The training process of DBNs generally includes two stages:

Stage1: For $i=1$ to 2 \{Train parameters for layer $i$ and $i+1$ using RBM.\};

First, input the training data $v=\left(v_{1}, v_{2}, v_{3}, \ldots, v_{m}\right)$ to RBM, and train the weight coefficient matrix $w_{1}$ of the first layer RBM on the basis of the formula $\Delta w_{i j}=\varepsilon\left(<v_{0} h_{0}>-<v_{1} h_{1}>\right)$. Second, fix $w_{1}$, train hidden vector $h^{1}$ according to the formula (2), regard $h^{1}$ as the input of the second layer RBM; Third, repeat the above operation, until the calculation of $h$ and ${ }^{w}$ are finished.

Stage 2: Fine-tune all the parameters using back propagating error derivatives.

After the completion of the RBM stack up, the output of the original training is used as the supervised data to fine-tune the parameters $\theta=(w, b)$ on each layer top-down, and makes the DBN achieve the global optimum. After the completion of the training, the vector will be used as the input of the FB algorithm. 


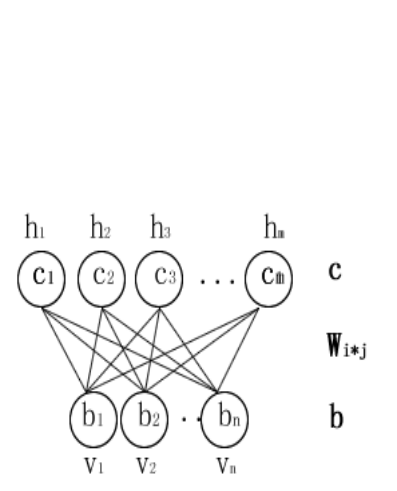

Fig.2 RBM

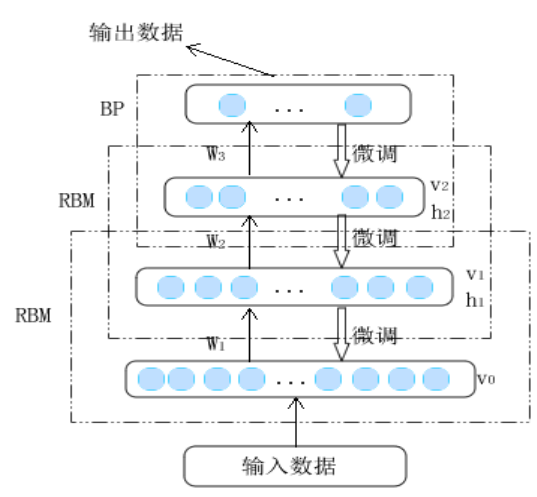

Fig.3 DBN framework

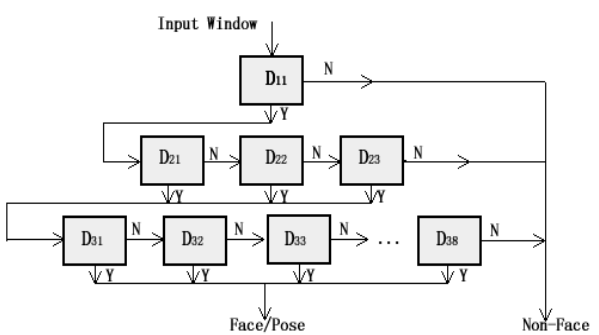

Fig.4 detector-pyramid architecture

In the classification stage, use detector-pyramid structure(see for fig.4) cascaded by 12 detectors as classifier, it divides face poses from left to right, coarse to fine into 12 kinds of scopes to better avoid missing faces in the image and each kind of scope corresponds to a detector. In the structure, $D_{L V}$ denotes a detector which detects face in the view range of $V$ at pyramid level $L$, the structure has three layers, the one, on the top is for the coarsest faces classification in the whole range of out-of-plane rotation; On the upper basis, the second layer divide face ranges into three groups:[-90, -30], [-30, 30],[30,90], and the third layer continues to divides head rotation scope in accordance with the rotation change of 20 degree. Learned from the paper [1], the change of rotation angle range in $[-90,-60]$ and $[60,90]$ are not just evident, so we regard them as one individual group independently, thus the number of detectors in the third layer is only 8: $[-90,-60],[-60,-40],[-40,20],[20,0],[0,20]$, [20,40], [40,60], [60, 90]. Therefore, there are 12 detectors in this classifier. There are only 7 of them need samples to train, while the rest can be obtained by using mirror image method on the basis of the symmetry of face. The training process of the detector is carried out by FB algorithm, which is put forward for the purse of minimizing error in the algorithm. The detailed algorithm steps of FB can be referred from article [1].

This method avoids too much manual intervention and provides a better solution to detect faces under unconstrained. Use pixel level face image as input of DBN, can learn the abstract feature which is implicit in the image. Furthermore, because the image is pre processed in advance, this method not only overcome the defect that when the input feature is the vector, the DBN cannot learn the local structure feature of face image well, but enhance the learning ability of the deep network. It is difficult using softmax algorithm to classify when head rotation changes slightly. While FB algorithm has rotation invariance, and can be used to successfully deal with the image when each kind of labels is not mutual exclusive. Two methods are combined to effectively reduce the sensitivity to the conditional transformation. The processes of the proposed algorithm are:

1. First, pre process the image according to three steps: grayscale image, histogram equalization and brightness normalization;

2. Second, input the image (namely training sample $\left.v=\left(v_{1}, v_{2}, v_{3}, \ldots, v_{m}\right)\right)$ obtained from 1 into DBN model at pixel level, get $m$ dimensions sample $Y=\left(y_{1}, y_{2}, y_{3}, \ldots, y_{n}\right)$, which can also be considered as $n$ characteristics of the sample.

3. Third, input $Y=\left(y_{1}, y_{2}, y_{3}, \ldots, y_{n}\right)$ into FB algorithm, and then train the algorithm to get a set $\mathrm{H}_{m}=\left\{h_{1}, \ldots, h_{m}\right\}$, which is integrated by $m$ optimal weak classifiers.

4. Combine $m$ weak classifiers $h_{m}(y)$ to a strong classifier $H_{M}(y)$ linearly, the combination formula is: $H_{M}(y)=\sum_{m=1}^{M} \varrho_{m}(y)+M T$. Where $T=\frac{1}{2} \log \frac{p(t=+1)}{p(t=-1)}$ is used to balance detection rate and false alarm rate, $\mathscr{E}_{m} \%(y)=\frac{1}{2} \log \frac{p\left(y_{m} \mid t=+1, w^{m-1}\right)}{p\left(y_{m} \mid t=-1, w^{m-1}\right)}, y$ is the subset of sample $Y, t=+1$ denotes that $y$ is visual, otherwise $\mathrm{y}$ is invisible. $M$, which denotes the total steps of the learning, is an integer. $w$ is weight, in the learning process, its value is updated after each iteration.

5. Finally, 10 strong classifiers are used as a detector to build a detector pyramid to carry out face 
detection. The detection results $s$ are labeled with a red border.

\section{Experiment result and analysis}

The main purpose of this paper is to implement multi-view and partial occlusion face detection under unconstrained environment. The algorithm is tested in Matlab R2011b software environment on an Intel 2.5GHz Dual Core PC with 4GB memory. The experiment data from the standard face database LFW and collected dataset, which contains face in occlusion, poses etc. that appropriately reflect the actual environment, are taken in outdoors. Select 900 images (which contain 1351 human faces) from LFW and 300 images (which contain 406 human faces) from collected dataset randomly, and then select $10 \%$ of the dataset as training sample, the rest as testing sample. DBN with 18 hidden units used in this paper has 3 layers, the learning rate in each layer is 0.002, 0.003and 0.005 respectively. The number of iteration is 40 in each layer. Each detector in the pyramid structure is composed of approximately 10 strong classifiers. Here are the experiment results of this method on the LFW and the collection dataset:

Table1 the detection results of our proposed algorithm

\begin{tabular}{lcccc}
\hline library & Number of detection & Detection rate/\% & false alarm & missing number \\
\hline LFW & 1348 & 99.78 & 23 & 26 \\
$\begin{array}{l}\text { collected } \\
\text { data }\end{array}$ & 405 & 99.75 & 15 & 16 \\
\hline
\end{tabular}

The experiment result on LFW is better than that on collected data. Because the background of the collected data is more sophisticated, face poses are more diverse and face ornaments are more casual. All of these will increase the difficulties on face detection. Compare our algorithm with LBP+DBN and DBN based on the article [6] to verify the superiority of this algorithm. The classification algorithm in the latter two algorithms is softmax. The experiment environment and dataset in the three algorithms are completely consistent. Select 10 face images whose face numbers are greater than or equal to 15 and resolution ratio is $560 \times 394$ from collected data. Select 5 images of them as training sample, the rest as testing sample. The ROC curves of these three contrast algorithms are shown in Fig. 5.

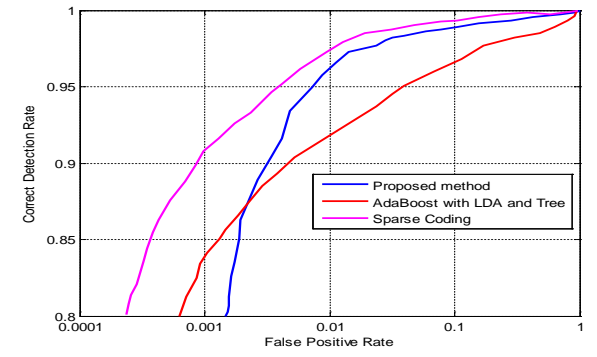

(a) Comparison of Detection rate and false Positive rate

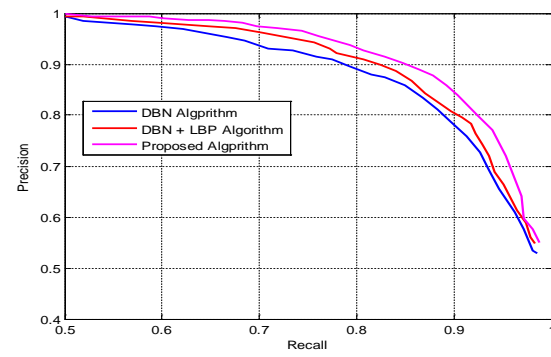

(b) Comparison of Precision and Recall

Fig. 5 ROC curve

Table2 Results of our algorithm and traditional algorithm

\begin{tabular}{cccc}
\hline Index & DBethod & LBP + DBN & The proposed algorithm \\
\hline Detection rate & $80.94 \%$ & $92.32 \%$ & $96.57 \%$ \\
Precision & $76.03 \%$ & $84.08 \%$ & $90.98 \%$ \\
\hline
\end{tabular}

The upper table and figures illustrate that the accuracy of this method is higher than other two algorithms' and the false positive rate of the proposed method is lower than that of the rest under the same experiment conditions. The detection rate of this algorithm is increased by $4.25 \%$ and $15.63 \%$ respectively compared to DBN and $\mathrm{LBP}+\mathrm{DBN}$. This is because when features go through the detector, they pass three levels, and each layer plays a role on filtering false positives. The top level can filter out $60 \%$ of the non face sample, and retain $99.98 \%$ of face sample; on the upper basis, the middle level can filter out $97 \%$ of the non face image, keep $99.53 \%$ sample from the upper left; while the bottom level can filter out $99 \%$ of non face image, and remain $99.26 \%$ face sample. Compared 
with the traditional DBNs model, our model based on detector-pyramid structure can accurately detect multi-view face is mainly because that the structure divides facial pose variation range delicately. Only 7 detectors need to be trained according to the symmetry of face and the speed of FB algorithm itself is fast, the whole training time of the system reduces greatly. Seen from the ROC curve in the fig.5 and the table 2, pyramid type of classifier not only does not make the learning ability of the whole system and its training speed decrease, but make the performance of the whole detection model improve to some extent. In this paper, the model with three layers has only eighteen hidden units, and the training process is not artificial but automatic, therefore, the training time and the fine-tune time is greatly reduced to meet the practical application.

\section{Summary}

In this paper, we integrated detector-pyramid into DBN to carry out multi-view face detection. Use DNB to extract face features automatically on the basis of the relevance between each part on face, thus avoid too much manual intervention. Use floatboost algorithm, which overcomes the limitation that softmax can perform well only if the categorization labels are mutual exclusion to divide facial pose variation range delicately, and effectively solve the false alarms and missing caused by tiny range change on multi-view face detection. The experiment results reveal that the proposed algorithm overcomes the defect that the traditional algorithm cannot solve the nonlinear factors, and result in a good detection rate in various poses, illuminations and occlusions. But this method also has some limitations, for examples, its training time will be too long when the hidden units scale is too large etc. Therefore, how to improve the learning efficiency and the training time will be the next research focus in future.

\section{Acknowledgements}

This research is supported by the National Natural ScieceFoundation of China (No.61263019).

\section{References}

[1] S.Z. Li, Z.Q. Zhang, FloatBoost learning and statistical face detection, IEEE Transactions on Pattern Analysis and Machine Intelligence. 26(2004) 1112-1123.

[2] Jairath S, Bharadwaj S, Vatsa M, et al., Adaptive skin color model to improve video face detection, Machine Intelligence and Signal Processing, Springer India, 2016, pp. 131-142.

[3] G.E. Hinton, R. Salakhutdinov, Reducing the dimensionality of data with neural networks, Science, 313 (2006) 504-507.

[4] G.E. Hinton, S.Osindero, Y.W.Teh, A Fast Learning Algorithm For Deep Belief Nets, Neural Computation, 18(2006) 1527 - 1554.

[5] R.D. Hjelm, V.D. Calhoun, R. Salakhutdinov et al., Restricted Boltzmann machines for neuroimaging: an application in identifying intrinsic networks, Neuroimage. 2014, 96, pp. 245-260.

[6] S.F. Liang, Y.H. Liu, L.I. Li-Chen, Face recognition under unconstrained based on LBP and deep learning, Journal on Communications, 35( 2014)154-160. 